\title{
The Optimization Model and Algorithm for Train Connection at Transfer Stations in Urban Rail Transit Network
}

\author{
Li Xuan ${ }^{1,2, *}$ and Shi Yang ${ }^{3}$
}

${ }^{1}$ School of Maritime and Transportation, Ningbo University, Ningbo, Zhejiang, 315211, P.R. China; ${ }^{2}$ National Traffic Management Engineering \& Technology Research Centre Ningbo University Sub-centre, Ningbo, Zhejiang, 315211, P.R. China; ${ }^{3}$ Ningbo Urban Planning \& Design Institute, Ningbo, Zhejiang, 315042, P.R. China

\begin{abstract}
In urban rail transit network, the passenger transfer time depends on the train connection states in transfer stations, therefore, the optimization of the connection relations of arrival and departure time among trains is significant to improve the level of transfer service. Here, with the psychology of waiting passengers taken into consideration, the cost function of transfer waiting times has been established. On this basis, an optimization model for train connections at transfer stations was constructed, and a genetic algorithm was designed to solve this model. A computer program implementing this genetic algorithm was written in Microsoft VB.NET. This program was used to optimize the train operation plans of a simple network which consists of four urban rail lines in Beijing. The results show that the proposed method can effectively reduce the total waiting time cost of all transfer passengers in the network.
\end{abstract}

Keywords: Genetic algorithm, Train connection optimization, Transfer, Urban rail transit network.

\section{INTRODUCTION}

With the expansion of the urban rail transit network, the number of transfer nodes on the travelling routes of passenger has increased substantially, and transfer has become a necessary step in most rail travels. To provide safe and efficient transfer service is key to improving the overall service level of the rail transit network. The time required for transfer is a direct manifestation of transfer efficiency, and it also directly reflects the level of the rail network management. Previous studies on rail transfers have focused largely on transfer station design and on transfer stream design in order to minimize the passengers' walking time during transfer. However, restricted by a variety of objective conditions, the room for reducing the transfer walking time is rather limited. In addition, compared to reducing the walking time, reducing the transfer waiting time is more valuable to passengers. The transfer waiting time is directly associated with the arrival and departure time of trains recorded before and after transfer at the transfer station. Thus, coordinating and optimizing the schedules of trains in different lines connected by transfer stations can make optimal connection between the arrival and departure time of trains, thereby successfully reducing the transfer waiting time of passengers at the program level.

At present, in most major cities, the train schedules are compiled using the method of "making schedules by line and adjusting schedules at the network level." More accurately, the schedules are first made at the level of individual lines in accordance with the passengers' demand and transport capacity resources; train schedules of different lines are compiled

*Address correspondence to this author at the School of Maritime and Transportation, Zhejiang, Ningbo 315211, P.R. China; Tel: 13857884371; E-mail: lixuan423@126.com separately. Then, at the network level, adjustments are made from the perspective of coordination and overall benefits. However, with this method, once the train schedules are completed, it is difficult to change factors, such as the number of running trains, intervals and dwelling times, and adjustments can only be made within a very narrow range . Furthermore, because of the complex coupling relationships between the lines, the complexity increases as the network scale expands, and the adjustment in a single point exerts time propagation effects on the entire network, which further limits the impact of the adjustment. Therefore, it is believed that the current method of compiling train schedules does not take into account the collaborative relationship between the lines, directly leading to an increase in passenger waiting time in the transfer stations within the network. This has created a bottleneck at the service level of the rail transit network.

Based on the existing results and issues, this paper treated the train system as a black box and used global coordination as the guideline to develop an optimization model for train connections at transfer stations. The goal is to achieve mutual coordination between the train schedules of different lines. The effectiveness of the optimization method has been verified using a specific example.

\section{LITERATURE REVIEW}

With the expansion of the urban public transport network, there has been continued growth in the proportion of time spent by passengers at the transfer nodes during their overall travel time. Therefore, the coordination and optimization of transit timetables (schedules) targeting the optimization of transfer waiting times have received wide attention from researchers. For a systematic review of the existing 
literature, the previous research was divided into three series according to the object of study, and then summarized. In the first series, transfer nodes in the regular bus system were the object of the study. In the second series, the object of study was the transfer hubs in the urban public transit system, consisting of regular bus and rail transit system. In the third series, the object of study was the transfer nodes in the urban rail transit system.

The first and second series investigated how to achieve efficient connections between buses in different lines or between the buses and trains at the transfer station via optimization of the bus timetables. The preparation of train timetables was different from that of bus timetables primarily due to the following reasons. First, rail transit was made limited by the train running intervals; also, the running time in this section, the dwelling time in the station and the departure interval were all relatively fixed, tolerating only small fluctuations. Methods for optimizing the bus timetables cannot be directly applied to rail transit. In addition, to improve the success rate of connections via real-time scheduling (holding at station, departure ahead of time, etc.) is well suited for regular bus transportation, yet it can hardly be applied to rail transit.

The third series explored connection optimization at the transfer stations between different lines within the urban rail transit system, using basis intrinsic characteristics of the urban rail transit system. This study belongs to the third series, and a brief literature review here will focus on research related to the third series. The number of published studies in the third series is relatively small, and these studies can be divided into three categories.

Studies in the first category optimized the schedules of multiple lines intersected in a single transfer station, but merely realized train connection optimization at one transfer station, without considering the overall optimization of multiple transfer stations within the network. For example, Partha (1995) [1] used a single transfer node as the object of study and set the objective to be a minimum of the sum of transfer passengers' waiting times and the initial waiting time of the arriving passengers at this node. With this method, a linear programming model was established, and a genetic algorithm was designed to solve the model. Ma (2010) [2] constructed models for the optimization of train timetables at the transfer stations in urban rail transit systems under the condition of transfers at the same platform and under the condition of transfers through tunnels, and designed a heuristic algorithm to design the model.

Studies in the second category established mathematical models using minimization of the total transfer time (cost) of all passengers in the rail transit system as the objective. On the basis of existing train schedules separately compiled by line, the running times, the dwelling times or turnaround times are adjusted to optimize the existing train schedules. To achieve this goal, Wong et al. (2008) [3] designed a heuristic search algorithm., Vansteenwegen $(2006,2007)$ [4, 5] designed a discrete event simulation method and Kwan et al. (2008) [6] designed a non-dominated sorting genetic algorithm.
For studies in the third category, a second-order coordination structure, namely "connection at transfer points coordination at the network," was used. First, inside each transfer node, the connection between the arrival and departure times of the trains was optimized, using methods similar to those in the second category of studies, i.e., on the basis of existing train schedules for separate lines, the train paths translation or allocation of buffer time was carried out for optimization. Next, at the network level, train connections between different transfer nodes were subjected to global optimization. To this end, Zhang et al. (2009) [7] designed a preference-oriented hierarchical loop coordination algorithm based on coordination levels. Fang (2010) [8] and Zhou et al. $(2011,2012)$ [9, 10] proposed a station-by-station adjustment method related to the importance of the transfer stations.

In summary, studies in the first category of the third series only optimized connections between the arrival and departure times of the trains at single transfer stations. With this method, the conflicts between the optimal train connection schedules for multiple transfer stations within the network would be inevitable, yet the first-category studies did not explore how to resolve these conflicts. In the studies of the second and third categories, the general method adopted was to make coordinative adjustment on the existing train schedules of individual lines prepared separately. As mentioned earlier, once the train schedule is completed, the number of running trains per line, running intervals, dwelling times and other factors are difficult to change; thus these adjustments can only be made within a very small range, and are not able to fully benefit the coordinated operation of the entire network. Moreover, to solve coordination between the transfer nodes in the network, studies in the third category artificially considered transfer stations or lines, thereby determining the priority in coordinative optimization between different transfer stations and lines. This provides a method for resolving the conflict between the train connection schedules for different transfer stations; however, it is too subjective and may generate closed-loop connections that have contradictions. More importantly, based on this method, the obtained network train schedules do not provide the optimal solution.

From the existing research results in this field, the following conclusions can be drawn. First, the current coordination of network transit timetables (operation charts) targeting the optimization of transfer connections mostly focused on the optimization of regular bus timetables. Yet vehicle management of regular buses is greatly different from that of rail transportation, and hence the method used for optimizing regular bus schedules cannot be directly applied to rail transit.

Secondly, the number of published studies on train connection optimization targeting urban rail transit networks is relatively small. To achieve the optimization of train connections at all transfer stations within the network, all train schedules must be prepared with the global network taken into consideration from the start, rather than merely making coordinative adjustments on the existing train timetables prepared separately by lines. This is the fundamental difference between the present study and all the previous studies. 
Meanwhile, the previous studies typically used minimization of the transfer wait times as the optimization objective, yet the transfer wait time was not entirely inversely proportional to passengers' satisfaction. In fact, it has been shown that when the waiting time is less than $30 \mathrm{~s}$, the degree of passenger dissatisfaction is highest [6]. Therefore, the present paper considered the psychology of waiting passengers and converted the transfer waiting time into the transfer cost. Minimization of the transfer cost was set as the objective to construct the optimization model for train connections at transfer stations in urban rail transit systems.

\section{ASSUMPTIONS}

This study is based on the following assumptions:

1. The transport configuration of all lines in the network can meet the passenger's demand, and the facilities and capacities of all transfer stations can also meet the passengers' demand.

2. The running times in the section, the dwelling times in the station, train running intervals and transfer walking times at all transfer stations are all known input parameters.

3. The passenger transfer demand is relatively stable over a period of time.

4. After arriving at the platform for the connecting direction, all transfer passengers take the first available connecting train.

5. Transfer between the up-bound and down-bound directions in the same line is not taken into consideration.

6. There is no joint operation between the lines, and for lines with multiple routes, only one route is considered.

7. The isolated lines in the network are not considered in the optimization model.

\section{THE ESTABLISHMENT OF THE OPTIMIZATION MODEL}

\subsection{Problem Analysis}

The basic train operation data, such as running time in the section, dwelling time in the station, and train running intervals are determined and the train schedule of one certain line in the coordination time period is determined by the departure time of the first train in this time period. Let the coordination time period be $\left[t_{0}, t_{1}\right]$, the departure time of the first train is at time point $[0, h)(h$ is the train running interval) after the starting time point of the coordination period $t_{0}$. In other words, the time interval between the departure time of the first train and $t_{0}$ is variable in $[0, h)$.

Under the assumption that there is no joint operation between the lines, the train schedules of different lines are independent of each other. Because loop routing is usually used in urban rail transit systems, the train schedules of upbound train and down-bound train of the same line are close- ly associated with each other. For each line, after the train schedule in one direction within the coordination time period is set, the schedule of the trains in the other direction is also determined. Hence, in this paper the objects of train connection optimization were restricted to one direction of each line in the network; this direction is called the coordination direction. In this way, the decision variables of the optimization model become the time intervals between $t_{0}$ and the departure time of the first train of various lines coordination direction within the network in $\left[t_{0}, t_{1}\right]$.

The goal of train connection optimization is to achieve rational connections of trains from different lines at the transfer stations, thereby, reducing the cost of passengers' transfer waiting times. Therefore, the objective function of optimization model is the minimization of the total cost of passengers' transfer waiting times at all transfer stations in the network.

\subsection{The Cost Function Corresponding to the Transfer Waiting Time}

The transfer waiting time is the time interval between the point when the passenger arrives at the platform of the connecting line and the point when the passenger boards the connecting train. If the connecting train arrives just when the passenger arrives at the platform of the connecting line, the passenger's transfer waiting time is 0 ; if the connecting train has just departed, the passenger's transfer waiting time is close to the difference between the running interval of the connecting train and time when the connecting train stops at this particular transfer station. These are the two extremes of the transfer status. The latter is the worst scenario for the passenger, because the transfer waiting time is the longest; however, the former scenario is not ideal either. A survey has shown that when the transfer waiting time is less than $30 \mathrm{~s}$, the dissatisfaction degree of transfer passenger is rather high [6]. The main reason for this is that a fixed connection time creates psychological pressure of a potentially missed connection on transfer passengers. This crisis is particularly evident when the train running frequency is low. Thus, this paper introduced the concept of a comfortable passenger waiting time, which is the waiting time that satisfies the passenger's psychological comfort. The optimal transfer status refers to a situation in which the transfer waiting time exactly equals the waiting time comfortable for the passenger.

Next, the cost function $C(t)$ corresponding to the transfer waiting time will be established. The cost corresponding to the waiting time comfortable for passengers is minimum; the cost is increased when the transfer waiting time is greater or smaller than the comfortable passenger waiting time. Thus the following function is used (function plot shown in Fig. (1):

$$
C(t)=\left\{\begin{array}{lc}
C_{1}-\frac{C_{1}}{R T} \times t, & t<R T ; \\
\frac{C_{2}}{h_{c o n}-D T_{c o n}^{a}-R T} \times(t-R T), & t \geq R T .
\end{array}\right.
$$


$t$--- transfer waiting time, $\min$;

$R T$--- comfortable passenger waiting time, min;

$h_{c o n}$--- running interval of the connecting train, min;

$D T_{\text {con }}^{a}$---the time when the connecting train stops at the transfer station $a$, min;

$C_{1}, C_{2}$---coefficients.

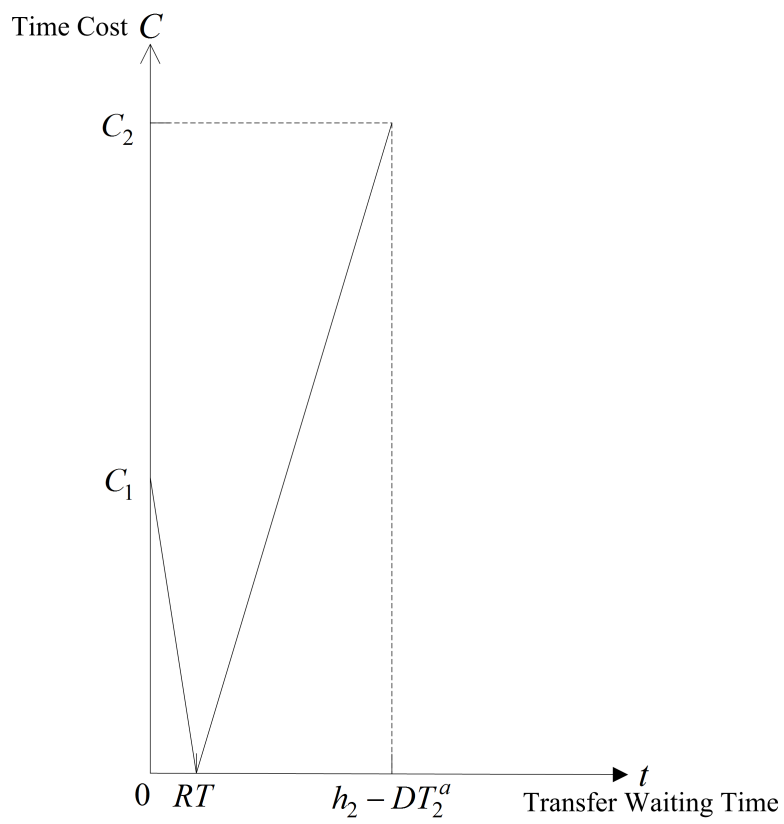

Fig. (1). The cost function phase of transfer waiting time.

The ideal transfer status can be observed when the passenger arrives at the platform of the connecting line, and the connecting train is about to arrive at the same station. The operation time from the train arriving at a station to a full stop is about $40 \mathrm{~s}$. Hence, here the comfortable passenger waiting time $R T$ is recommended to be $0.67 \mathrm{~min}$.

The values of coefficients $C_{1}$ and $C_{2}$ can be determined by the passenger travel time value. The waiting time of 0 brings the passenger a certain psychological stress, which is equivalent to the generation of a certain time cost, i.e. $C_{1}$.

Here the value of the time the passenger spends on waiting after having boarded the train is used to determine the value of coefficient $C_{1}$. Parameter $C_{2}$ describes the cost of waiting time when the passenger misses the expected connecting train.

In public transportation, the value of the waiting time is often determined relative to the value of the riding time [5]. The per unit riding time value is set to 1 , denoting the value of unit time that the passenger spends on the running train. Then, the per unit value of time that the passenger spends on the platform waiting for the train is set to 2.5 , meaning that the time cost of riding for $2.5 \mathrm{~min}$ is equivalent to that of waiting on the platform for $1 \mathrm{~min}$. If the worst transfer status is observed, the passenger will wait for a period of about $h_{\text {con }}-D T_{\text {con }}^{a}$. In this case, the psychological anxiety of waiting is enhanced, leading to increased time value; the per unit waiting time value in this case is set to 2.7 [5]. Compared to waiting on the platform, a passenger waiting inside the train is more comfortable, and the value of unit time spent on waiting in the train is set to 2 . Thus, $C_{1}=2 D T_{\text {con }}^{a}$ and $C_{2}=2.7\left(h_{c o n}-D T_{c o n}^{a}\right)$ were set in the study.

\subsection{Mathematical Model}

According to the above analyses, targeting the coordination time period $\left[t_{0}, t_{1}\right]$, the following optimization model for train connections at transfer stations in urban rail transit networks was constructed:

$$
\begin{aligned}
& \min F(X)=\sum_{i=1}^{m} \sum_{j=1}^{r_{i}} \sum_{s=1}^{g_{i, j}} C\left(t_{i, j, s}(X)\right) \times V_{i, j, s} \\
& \text { s.t. } X=\left(x_{1}, x_{2}, \mathrm{~L}, x_{n}\right) x_{k} \in\left[0 h_{k}-1\right] \text { and } x_{k} \in N \quad 1 \leq k \leq n \\
& C\left(t_{i, j, s}(X)\right)=\left\{\begin{array}{l}
2 D T_{c o n}^{i, j}\left[1-\frac{t_{i, j, s}(X)}{R T}\right], \\
2.7 \times\left(1+\frac{R T}{h_{c o n}^{i, j}-D T_{c o n}^{i, j}-R T}\right) \times\left(t_{i, j, s}(X)-R T\right), t_{i, j, s}(X) \geq R T .
\end{array}\right.
\end{aligned}
$$

Where,

$X$ is the decision variable. It is an $n$-dimensional vector and $n$ is the number of lines in the network (isolated lines not included);

$x_{k}(1 \leq k \leq n)$ is the time interval between $t_{0}$ and the departure time of the first train of line $k$ 's coordination direction in the time period $\left[t_{0}, t_{1}\right]$, and $x_{k}$ is a natural number in the interval $\left[\begin{array}{ll}0 & h_{k}-1\end{array}\right], \min$;

$h_{k}$ is the running interval of line $k, \min$;

$t_{i, j, s}(X)$ is the transfer waiting time of the $s$ th batch of transfer passengers in the $j$ th transfer connection at the $i$ th transfer station in the time period $\left[t_{0}, t_{1}\right]$. Under the network train schedules corresponding to $X(\mathrm{~min})$, a transfer connection describes a transfer from up-bound/down-bound of a feeder line to the up-bound/down-bound of a connecting line

$C\left(t_{i, j, s}(X)\right)$---the cost function corresponding to the transfer waiting time $t_{i, j, s}(X)$;

$D T_{c o n}^{i, j}$---the time when the connecting train stops in the $j$ th transfer connection at the $i$ th transfer station;

$h_{c o n}^{i, j}$---the running interval of the connecting train in the $j$ th transfer connection at the $i$ th transfer station;

$V_{i, j, s}$---the total number of the $s$ th batch of transfer passengers in the $j$ th transfer connection at the $i$ th transfer 
station in the time period $\left[t_{0}, t_{1}\right]$. It is estimated according to the statistics of passenger volume in this time period at the transfer station, and based on the assumption that within the time window of statistics, the number of passengers in different batches wasthe same;

$m$---the number of transfer stations in the network;

$r_{i}$---the number of transfer connections at the $i$ th transfer station; $r_{i}=\left(2 n_{i}^{t}+n_{i}^{e}\right)^{2}-\left(4 n_{i}^{t}+n_{i}^{e}\right)$, where $n_{i}^{t}$ and $n_{i}^{e}$ represent the number of lines passing through and ending at the $i$ th transfer station.

$g_{i, j}$---the number of batches of passengers in the $j$ th transfer connection at the $i$ th transfer station in the time period $\left[t_{0}, t_{1}\right] ; g_{i, j}=\frac{t_{1}-t_{0}}{h_{f e e}^{i, j}}$, where $h_{f e e}^{i, j}$ represents the running interval of the feeder train in the $j$ th transfer connection at the $i$ th transfer station.

\section{DESIGN OF THE OPTIMIZATION ALGORITHM}

\subsection{Methods for Solving the Optimization Model}

In the optimization model, it is difficult to express mapping from the decision variable $X$ to the transfer wait time $t_{i, j, s}(X): \quad X \rightarrow t_{i, j, s}(X)$ using mathematical formulas. Therefore, the model cannot be solved with traditional analytical methods.

Meanwhile, different element combinations of $x_{k}$ ( $1 \leq k \leq n$ ) form the feasible region of $X$, and the number of feasible solutions is expressed as $\prod_{k=1}^{n} h_{k}$. This paper considered the currently operating Shanghai rail transit network as an example. It contains 14 lines. In the simplest scenario, it was assumed that the running intervals of all lines in the coordination time period were $5 \mathrm{~min}$, then the number of feasible solutions reached $5^{14}=6103515625$. Thus, it is difficult to simply apply an exhaustive search method to find the optimal solution. A heuristic algorithm is most commonly used to solve this type of problem. Here a genetic algorithm has been used to solve the optimization model.

\subsection{The Steps of the Genetic Algorithm}

The overall steps of the genetic algorithm are as follows:

1. The initial population is randomly generated to obtain the first generation of individuals. Each individual is represented as gene encoding of the chromosome.

2. The fitness of each individual is calculated.

3. The fitness, gene encoding and function value of the individual with the maximum fitness in the current generation are all recorded.
4. It is determined whether the condition for stopping evolution is satisfied; if yes, then the calculation is stopped here, otherwise the evolution continues.

5. Regenerating individuals are selected according to fitness. Individuals with high fitness have a high probability of being selected, whereas those with low fitness are likely to be eliminated.

6. According to certain crossover probabilities and crossover methods, new individuals are generated.

7. According to certain mutation probabilities and mutation methods, new individuals are generated. The new generation of population is thus obtained, and the loop returns to step 2 .

\subsection{The Key to the Design of the Genetic Algorithm}

\subsubsection{Encoding and Decoding}

The decision variable $X$ in the optimization model is an integer vector, and can be viewed as the phenotype of the genetic algorithm. The mapping process from the phenotype to the genotype involves encoding. This paper used binary encoding to represent the individual's genotype. The specific encoding and decoding methods are as follows.

One chromosome represents one combination of the intervals between $t_{0}$ and the departure time of the first train of $n$ lines in $\left[t_{0}, t_{1}\right]$.

Each chromosome can be divided into $n$ segments; the bit string in the $k$ th $(1 \leq k \leq n)$ segment represents the time interval between $t_{0}$ and the departure time of the first train of line $k$ 's coordination direction in $\left[t_{0}, t_{1}\right]$. To encode the bit string in the $k$ th segment, a binary string encoding method for integer is used. Let integer $x_{k} \in\left[0, h_{k}-1\right](1 \leq k \leq n$ ). $h_{k}-1$ is dissociated using the following algorithm:

$$
\begin{aligned}
& n_{0}=0 ; x=h_{k}-1 ; p=0 \\
& \text { do } p=p+1 \\
& \quad n_{p}=\operatorname{int}\left[\log _{2}(x+1)\right] \\
& \quad x=x-2^{n_{p}}+1 \text { while } x>0
\end{aligned}
$$

The $p$ integers $n_{j}(j=1,2, \mathrm{~L}, p)$ generated from the above algorithm satisfies $h_{k}-1=\sum_{j=1}^{p}\left(2^{n_{j}}-1\right)$. Namely, $h_{k}-1$ is the sum of $p$ binary numbers with digit number $n_{j}(j=1,2, \mathrm{~L}, p)$. Thus, $x_{k}$ can be represented by $\left(n_{1}+n_{2}+\mathrm{L}+n_{p}\right)$ binary strings $B_{k}$, i.e., $\quad x_{k}=\sum_{j=1}^{p} B_{k}^{j}=$ $\sum_{j=1}^{p}\left(b_{n_{j}-1}^{j} b_{n_{j}-2}^{j} \mathrm{~L} b_{0}^{j}\right)$, where, $b$ is the binary bit of 0 or 1. 
For example, if $x_{1} \in[0,5]$, after decomposing $h_{1}-1=5$ , three integers $\left\{n_{1}=2, n_{2}=1, n_{3}=1\right\}$ are obtained, which satisfy $5=\left(2^{2}-1\right)+\left(2^{1}-1\right)+\left(2^{1}-1\right)$. Thus, $x_{1}$ can be represented by four-digit binary strings $B_{1}=\left(b_{1}^{1} b_{0}^{1}\right)_{1}\left(b_{0}^{2}\right)_{1}\left(b_{0}^{3}\right)_{1}$, namely $x_{1}=\sum_{j=1}^{3} B_{1}^{j}=\sum_{j=1}^{3}\left(b_{n_{j}-1}^{j} b_{n_{j}-2}^{j} \mathrm{~L} b_{0}^{j}\right)_{1}$.

From the above encoding method, the chromosome decoding method can be obtained. First, the chromosome is divided into $n$ segments. For $\forall k(k=1,2, \mathrm{~L}, n)$ segment, the binary digits $\left(n_{1}+n_{2}+\mathrm{L}+n_{p}\right)$ are determined, and the $p$ binary numbers with the number of digits $n_{j}(j=1,2, \mathrm{~L}, p)$ are then converted into decimal numbers. Finally, the decimal numbers are added up to obtain $x_{k}$.

For example, this paper considered an urban rail transit network that contains four lines; the coordination time period was11:00-12:00, and the running intervals of the four lines in the time period were $6 \mathrm{~min}, 7 \mathrm{~min}, 5 \mathrm{~min}$ and $7 \mathrm{~min}$. Chromosome "110111101010110" provided a feasible solution. It was divided into 4 segments, and the number of digits in the different segments was $4,4,3$ and 4 . Then the binary number in each segment was dissociated to obtain "11 $|0|$ $1\|11|10\|10|1 \| 01| 10 . "$ Then all the binary numbers were converted into decimal numbers and added together, obtaining $x_{1}=4, x_{2}=5, x_{3}=3, x_{4}=3$. Thus the departure timings of the first train in the coordination direction entering the coordination time period were 11:04, 11:05, 11:03 and 11:03.

\subsubsection{Constructing the Fitness Function}

The fitness function is normally derived from the objective function, and needs to meet the basic condition of being single-valued, continuous, non-negative and maximized [11]. Considering that the optimization model finds the minimum value, and the value range of the objective function is $(0,+\infty)$, the fitness function can be set as the inverse of the objective function, namely:

$$
\text { Fit }(F(X))=[F(X)]^{-1}
$$

The smaller the value of the objective function, the larger is the corresponding fitness value, and the greater the probability of the individual gene to be passed on to the next generation. In addition, because the value of the objective function is generally not close to 0 , the fitness value determined by formula (5) does not show positive spillover. In the process of calculating the objective function value according to the phenotype of an individual with a certain chromosome, two steps are needed.

The first step is to determine the network of train schedules based on the decision variable $X$ and the known basic operation data of the trains. Two rules that need to be followed during the calculation process are clarified as follows.
First, if the locomotive use cycle is not an integer multiple of train running intervals, the locomotive operation cycle is enlarged by increasing the reentry time, so that the operation cycle becomes an integer multiple of the running intervals. In this way, the train running intervals within the coordination time period remain consistent.

Secondly, given one solution of $X=\left(x_{1}, x_{2}, \mathrm{~L}, x_{n}\right)$, from $x_{k}$ it can be observed that the departure time of the first train of line $k$ 's coordination direction in $\left[t_{0}, t_{1}\right]$ is $t_{k 1}=t_{0}+x_{k}$. It can then be deduced that the departure time of the first train of line $k$ 's non-coordination direction in $\left[t_{0}, t_{1}\right]$ is:

$t_{k 1}^{\prime}=t_{0}+x_{k}+t_{\text {travel }}^{k}+t_{\text {reentry }}-r \times h_{k}$

Where,

$t_{\text {travel }}^{k}$---the travel time of the train of line $k$ 's coordination direction;

$t_{\text {reentry }}{ }^{---}$the reentry time of trains of line $k$ 's coordination direction; $h_{k}$ denotes the train running intervals of line $k$ $; r=\left\lceil\frac{t_{0}+x_{k}+t_{\text {travel }}^{k}+t_{\text {reentry }}}{h_{k}}\right\rceil$, representing the greatest positive integer smaller than $\frac{t_{0}+x_{k}+t_{\text {travel }}^{k}+t_{\text {reentry }}}{h_{k}}$.

The second step is to match the train pairs for each transfer relation at all the transfer stations based on the network train schedules, and to calculate the transfer waiting time of the passengers for each train pair. Next, the corresponding time cost is calculated, and finally all time costs are added to obtain the objective function value $F(X)$.

\subsubsection{Conditions for Stopping the Loop}

In this paper, the following three conditions were used to determine if the loop should be stopped.

1. The optimal solution remained unchanged in the generation $G N_{\text {min }}$.

2. In one generation, the difference between the best fitness and the worst fitness was smaller than $\tilde{F}(\%)$.

3. The greatest number of evolving generations was $G N_{\max }$

\subsubsection{The Selection, Crossover and Mutation Operators}

\subsubsection{The Selection Operator}

In this study, fitness proportionate selection was used, combined with tournament selection. Fitness proportionate selection is the most basic selection method. The expected number of selections for each individual is associated with the ratio of its fitness value to the mean fitness value of the population. This method is similar to the roulette wheel in a casino. In tournament selection, before executing the selection operation, the individual with the highest fitness value in the parent population is selected as the global best individu- 


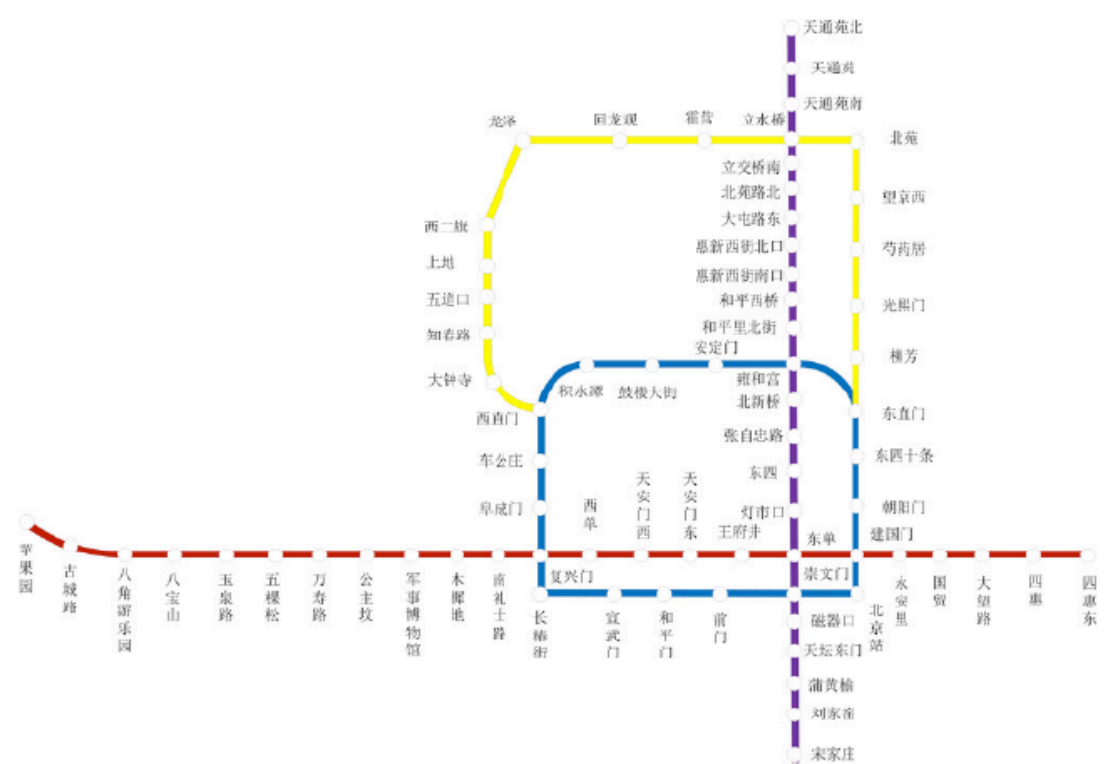

Fig. (2). Partial map of the beijing rail transit network.

al. After completing the selection and crossover operations, the best individual of the current population is selected as the local best individual who is then compared to the global best individual. The individual with the higher fitness value is then selected as the current global best individual. After one genetic operation is completed, the current global best individual is used to replace the individual with the lowest fitness value in the offspring generation.

\subsubsection{The Crossover Operator}

In this paper, uniform crossover was used in which each location on the chromosome bit string was subjected to random uniform crossovers with the same probability. The specific steps are as follows. For one pair of parent individuals, whether the crossover operation needs to be executed is determined according to the crossover probability $p_{c}$. If yes, a $0-1$ mask of the same length as the individual is randomly generated, and various segments of the mask determine which parent provides value for the offspring individual in the corresponding segments, and a new individual is thus generated.

\subsubsection{The Mutation Operator}

The mutation operator is implemented by randomly reversing the binary string of a certain allele according to the mutation probability $p_{m}$. Specifically, for a given chromosome bit string, $s=a_{1} a_{2} \mathrm{~L} a_{L}$, the operation is:

$a_{i}^{\prime}=\left\{\begin{array}{ll}1-a_{i}, & \text { if } x_{i} \leq p_{m} \\ a_{i}, & \text { otherwise }\end{array} \quad i \in\{1,2, \mathrm{~L}, L\}\right.$.

In this way, the new individual $s^{\prime}=a_{1}^{\prime} a_{2}^{\prime} \mathrm{L} a_{L}^{\prime}$ is generated. $x_{i}$ is the uniform random variable corresponding to each gene locus, $x_{i} \in[0,1]$.

\section{EXAMPLE}

To verify the effectiveness of the model and the algorithm, in the Win7 operating system, a program implementing the genetic algorithm was written in Microsoft VB.NET. Using this program, a simple network composed of Line 1, Line 2, Line 5 and Line 13 in Beijing rail transit system (as shown in Fig. 2) was subjected to the optimization of network train operation charts for a certain time period (11:0012:00).

The basic network data involved in the algorithm (including running times in the section, dwelling times in the station, train running intervals, reentry times, transfer walking times, the volume of transfer passengers, etc.) were set according to the actual operational data of the Beijing rail transit network. The running intervals for each line are shown in Table 1. The values of other relevant parameters are shown in Table 2.

Table 1. Train running intervals of each line (11:00-12:00).

\begin{tabular}{|c|c|}
\hline Rail Transit Line & Train Running Interval \\
\hline \hline No. 1 & $4 \mathrm{~min}$ \\
\hline No. 2 & $5 \mathrm{~min}$ \\
\hline No. 5 & $6 \mathrm{~min}$ \\
\hline No. 13 & $7 \mathrm{~min}$ \\
\hline
\end{tabular}

For all lines, the coordination direction is set to be downbound. The results obtained after running the program are shown in Table $\mathbf{3}$.

After optimization, the total cost of transfer wait times for all the passengers in this rail network from 11:00-12:00 was 30789.66. However, according to the current daily train 
Table 2. Parameter assignment for the genetic algorithm.

\begin{tabular}{|c|c|c|c|c|c|c|}
\hline$R T$ & $N$ & $p_{c}$ & $p_{m}$ & $G N_{\min }$ & $G N_{\max }$ & $F \%$ \\
\hline \hline $0.67 \mathrm{~min}$ & 50 & 0.5 & 0.01 & 50 & 200 & $1 \%$ \\
\hline
\end{tabular}

Table 3. Optimization results.

\begin{tabular}{|c|c|c|}
\hline \multirow{2}{*}{ No of Line } & Direction of Line & $\begin{array}{c}\text { The Departure Time of } \\
\text { the First Train }\end{array}$ \\
\hline \hline \multirow{2}{*}{ No.1 } & Up-bound & $11: 04: 00$ \\
\cline { 2 - 3 } & Down-bound & $11: 03: 00$ \\
\hline \multirow{2}{*}{ No.2 } & Up-bound & $11: 04: 00$ \\
\cline { 2 - 3 } & Down-bound & $11: 00: 00$ \\
\cline { 2 - 3 } & Up-bound & $11: 05: 10$ \\
\hline \multirow{2}{*}{ No.13 } & Down-bound & $11: 05: 00$ \\
\cline { 2 - 3 } & Up-bound & $11: 00: 30$ \\
\hline \multicolumn{2}{|c|}{ The Value of the Objective Function } & $11: 05: 00$ \\
\hline
\end{tabular}

schedules, the value of the objective function is 35633.23. Therefore, using the model and the algorithm proposed in the present paper for the optimization of the network train schedules, the value of the objective function was reduced by $13.59 \%$. Thus, it is proven that the method proposed in this study is effective.

Because this example only includes 4 lines, the number of feasible solutions is 360 ; it would be practical to apply the exhaustive search method to obtain the optimal solution. A program implementing the exhaustive search method was then written in Microsoft VB.NET. It was found that the optimal result obtained using the exhaustive search algorithm was the same as that obtained using the genetic algorithm. Hence it is proven that the genetic algorithm proposed in this paper is feasible and effective in solving the optimization model.

\section{CONCLUSION}

In order to improve the passenger service level of urban rail transit systems under the condition of network operation, the operation management department should coordinate and optimize the network train schedules according to the characteristics of passenger demands in the rail network, promoting rational connection between different lines at the transfer stations. This paper considered the psychology of passenger waiting into consideration and constructed the cost function of transfer wait times. On the basis of meeting the actual transfer passenger demand, this study proposed an optimization model for train connections at transfer stations in urban rail transit networks, so that benign interactions between the train flow and passenger flow can be established. In addition, a genetic algorithm was designed to solve the model. Finally, an actual rail network was used as an example to verify the effectiveness of the model and the algorithm. The proposed method can provide theoretical support and practical guidance for the optimized preparation of train schedules (timetables) in urban rail transit networks. This helped in solving the existing problem of the lack of coordination in the current preparation of train schedules, and promoted the formation of a good cooperative relationship between different lines in terms of passenger transfers within the rail network. In the future studies, we will consider train delays in order to improve the reliability of optimization results of train connection.

\section{CONFLICT OF INTEREST}

The authors confirm that this article content has no conflict of interest.

\section{ACKNOWLEDGEMENTS}

This work was financially supported by the National Natural Science Foundation of China (51408323) and Zhejiang Provincial Natural Science Foundation (LQ13G010010).

\section{REFERENCES}

[1] P. Chakroborty, K. Deb, P. S. Subrahmanyam, "Optimal scheduling of urban transit systems using genetic algorithms", Journal of Transportation Engineering, vol. 1, pp. 544-553, 1995.

[2] C.Y. Ma, Timetable coordination and optimization for transfer stations in urban rail transit, Beijing Transportation University, 2010.

[3] R.C.W. Wong, T. W.Y. Yuen, and K. W. Fung, "Optimal timetable synchronization for rail mass transit", Transportation Science, vol. 42, no. 1, pp. 57-69, 2008.

[4] P. Vansteenwegen and D. Van Oudheusden. "Developing railway timetables which guarantee a better service". European Journal of Operational Research, vol. 173, pp. 337-350, 2006.

[5] P. Vansteenwegen and D. Van Oudheusden. "Decreasing the passenger waiting time for an intercity rail network", Transportation Research Part B, vol. 41, pp. 478-492, 2007.

[6] C. M. Kwan, and C. S. Chang, "Timetable synchronization of mass rapid transit system using multiobjective evolutionary approach", IEEE Transactions on Systems, Man, and Cybernetcs-Part C: Applications and Reviews, vol. 38, no. 5, pp. 636-648, 2008.

[7] M. Zhang, and S.M. Du, "Transfer coordination optimization for network operation of urban rail transit based on hierarchical preference", Journal of the China Railway Society, vol. 31, pp. 9-14, 2009.

[8] X.H. Fang, Study on coordination theory and method of train operation on urban mass transit network, Beijing Transportation University, 2010.

[9] Y.F. Zhou, L.S. Zhou, and Y.X. Yue, "Synchronized and coordinated train connection optimization for transfer stations of urban rail networks", Journal of the China Railway Society, vol. 33, pp. 9-16, 2011. 
[10] Y.F. Zhou, Study on integration train timetabling theories and method for urban mass transit network, Beijing Transportation University, 2012.
[11] M.Q. Li, and J.S. Qou, Basic theories and application of the Genetic Algorithm, Science Press, Beijing, 2004.

Received: April 02, 2015

(C) Xuan and Yang; Licensee Bentham Open.

This is an open access article licensed under the terms of the Creative Commons Attribution Non-Commercial License (http://creativecommons.org/licenses/by-nc/4.0/) which permits unrestricted, non-commercial use, distribution and reproduction in any medium, provided the work is properly cited 